Check for updates

Cite this: RSC Adv., 2019, 9, 27096

\title{
Tannic acid-based nanopesticides coating with highly improved foliage adhesion to enhance foliar retention
}

\author{
Manli Yu, ${ }^{\text {ab }}$ Changjiao Sun, ${ }^{\text {bc }}$ Yumiao Xue, ${ }^{d}$ Chang Liu, ${ }^{d}$ Dewen Qiu, ${ }^{a}$ Bo Cui, ${ }^{b}$ \\ Yan Zhang, ${ }^{d}$ Haixin Cui (D) ${ }^{* b}$ and Zhanghua Zeng (iD *b
}

Poor utilization efficiency of conventional pesticide formulation has resulted in overuse, which could increase costs, toxicity to other non-target organisms, concerns about human health and safety, groundwater contamination, causing ecosystem destruction and food pollution. The folia-adhesive formulation is supposed to enhance foliar retention time and utilization efficiency. According to the microstructure of the foliage, the nanopesticides surfaces were modified by affinity groups to improve folia adhesion and decrease the loss from crop foliage. In this study, tannic acid, a bioadhesive natural molecule, has been applied to develop abamectin nanopesticide (Abam-PLA-Tannin-NS) and azoxystrobin nanopesticide (Azox-PLA-Tannin-NS) with strong adhesion to foliage by chemical modification. Abam-PLA-Tannin-NS and Azox-PLA-Tannin-NS presented better photostability and continuous release behavior. The retention rates of Abam-PLA-Tannin-NS and Azox-PLA-Tannin-NS on the foliage was remarkably enhanced by more than $50 \%$, compared with unmodified nanopesticides. Resultantly, the indoor toxicity of Abam-PLA-Tannin-NS and antifungal activity of Azox-PLA-Tannin-NS were enhanced. The interaction force between tannic acid coating nanoparticles and foliage was mainly from hydrogen bonding. Our findings could be beneficial to develop novel leaf-adhesive nanopesticides with high retention time and bioavailability.

Received 28th July 2019

Accepted 19th August 2019

DOI: $10.1039 / c 9 r a 05843 e$

rsc.li/rsc-advances

In the spraying process, most of the pesticide in traditional

\section{Introduction}

Pesticides are chemical or biological agents that deter, incapacitate, kill, or otherwise discourage pests to improve crop yield and quality by preventing crop losses to insects and other pests. ${ }^{1,2}$ They play a vital role in modern agriculture, providing substantial agronomic and economic benefits. However, resistance problems have increased, because pesticides are applied more frequently and at higher dosage rates. ${ }^{3-7}$ Reducing pesticide use and alternating among classes of pesticides with different modes of action can help to reduce the possibility of pest resistance. Managing pest resistance is very important in helping to prolong the effective period of pesticides. The overuse of pesticides brings several problems and limitations including increased costs, toxicity to other non-target organisms, concerns about human health and safety, groundwater contamination, and environmental and ecological quality. ${ }^{8-12}$

anstitute of Plant Protection, Chinese Academy of Agricultural Sciences, Beijing, 100193, PR China

${ }^{b}$ Institute of Environment and Sustainable Development in Agriculture, Chinese Academy of Agricultural Sciences, Beijing, 100081, PR China. E-mail: zengzhanghua@caas.cn; cuihaixin@caas.cn; Tel: +86-1082106004

${ }^{c}$ Gembloux Agro-Bio Tech, University of Liege, Belgium

${ }^{d}$ School of Materials Science \& Engineering, Beijing Institute of Fashion Technology, Beijing, 100029, PR China pesticide formulations fails to target the plant foliage, and the effective durable period on the crop foliage does not provide adequate pest control. ${ }^{13}$ The problems encountered during spraying include droplet drifting, jumping, rolling down, rain washing and decomposition, which can give rise to low effective utilization rate of pesticides $(<10 \%) .{ }^{14}$ Therefore, there is an urgent need to develop new approaches to control losses and improve the pesticide utilization efficiency. A new kind of pesticide formulation is required with advantages of high adhesion capacity on plants, low loss to the environment, long duration of efficacy, and low dosage and cost, so as to decrease the risk of environmental pollution, save manpower by reducing the application frequency, increase the safety of the pesticide user, and decrease the non-target effects when compared with traditional pesticides. ${ }^{15}$

Recently, the development of nano-pesticide formulations has been shown to improve pesticide performance by formulating nanoparticle-based delivery systems. ${ }^{16-25}$ The pesticide nano-delivery systems with small size and large surface area improved the deposition on foliage and extended the release time. Moreover, in terms of the crop foliage microstructure, the nanoparticles surface can easily be modified by affinity groups to improve adhesion and decrease the loss from crop foliage. ${ }^{15}$ In our previous study, the regulated adhesion to cucumber 
foliage was developed with different affinity groups by chemically modification. ${ }^{25}$ The low-cost natural adhesion product is more practical for nanopesticides, since chemical modification is more costly.

In nature, adhesive behaviors occur in many living creatures. ${ }^{26,27}$ Bioadhesive materials are one of the most promising materials for designing site-specific drug delivery systems. A naturally adhesive polydopamine-containing material inspired by mussels has been applied in many fields, in which catechol groups play a major role in adhesion to various surfaces. ${ }^{28-31}$ Polydopamine nanoparticles encapsulating pesticide exhibited excellent adhesive performance on crop foliage, and enhanced pesticide retention time. ${ }^{22,32}$ Given the high cost of dopamine, it is not practical for application as a pesticide carrier. Tannic acid (TA) is a natural polyphenol with low cost that can be extracted from various plants. It has valuable properties such as antioxidant, antibacterial, and biodegradability. ${ }^{33-35}$ TA has been widely applied in the generation of thin films or particles with adhesive properties because of its multitude of catechol groups, providing multiple bonding sites with diverse interactions, including hydrogen bonding, ionic bonding, coordinate bonding, and hydrophobic interactions. TA as adhesive agent has been rarely reported for pesticide formulation. TA coordinating with $\mathrm{Fe}$ (III) was used as shell to form pesticide microencapsulation, in which the role of TA as adhesive agent was not been discussed. ${ }^{36}$ Here, TA was applied to coat nanopesticides, and mechanism of the improvement on foliage adhesion and enhancement of foliar retention time were investigated in detail.

\section{Experimental section}

\section{Materials}

Abamectin (95.6\%) was purchased from Qilu Pharmaceutical Company, Ltd. (Shandong, China). Azoxystrobin (95\%) was supplied by Sheng Tianheng record Biological Technology Co., Ltd. (China). Poly(lactic acid) (PLA; MW approximately 100 000) was purchased from Daigang Biomaterial Company (Shandong, China). Poly(vinyl alcohol) (PVA) was purchased from Sigma Aldrich. Dichloromethane $\left(\mathrm{CH}_{2} \mathrm{Cl}_{2} ; 99.8 \%\right)$, TA (95\%) and poly(ethylene glycol) (PEG; MW approximately 10 000) were purchased from J\&K chemical company (Beijing, China). The water-dispersible granules (WDG) were purchased from Noposion Agrochemicals Co. Ltd. (Shenzhen, China). Commercial microcapsule and suspension concentrate (SC) were obtained from Zhengda Co., Ltd. (China). All chemicals were directly used as received. The water used in all experiments was Milli-Q water $(15 \mathrm{M} \Omega \mathrm{cm}$, TOC $\leq 4 \mathrm{ppb})$. Fusarium was supplied by the Beijing Academy of Agriculture and Forestry Sciences.

\section{Preparation of nanopesticides}

The Abam-PLA-Tannin-NS was synthesized by a classic solvent evaporation method. First, abamectin $\left(40 \mathrm{mg} \mathrm{ml}^{-1}\right)$ and PLA (40 $\mathrm{mg} \mathrm{ml}^{-1}$ ) were dissolved in $\mathrm{CH}_{2} \mathrm{Cl}_{2}$ by magnetic stirring. Second, the solution was added dropwise over 10 min into PVA aqueous solution (10 $\mathrm{mg} \mathrm{ml}^{-1}$ ) that was used as a surfactant by a shearing machine (C25, ATS Engineering Ltd., Vancouver, Canada) to emulsify, while being cooled in an ice-water bath to prevent the evaporation of $\mathrm{CH}_{2} \mathrm{Cl}_{2}$. Next, the mixed system was stirred vigorously $(1000 \mathrm{rpm})$ overnight to evaporate all the organic solvent at room temperature. Then, PEG $\left(12 \mathrm{mg} \mathrm{ml}^{-1}\right)$ was added to the nanosuspension and followed by dropping TA $\left(12 \mathrm{mg} \mathrm{ml}^{-1}\right)$. Finally, the nanosuspension was centrifuged at $15000 \mathrm{rpm}$ for $10 \mathrm{~min}$ at $4{ }^{\circ} \mathrm{C}$ and the collected pellet was redispersed in deionized water; this process was repeated three times to remove as much surfactant as possible. The nanosuspension was lyophilized by a freeze drier (FD-81, EYELA, Tokyo, Japan) to complete the preparation of Abam-PLATannin-NS (Scheme 1). Azox-PLA-Tannin-NS was prepared by following the same procedure.

\section{Determination of pesticide loading content}

The pesticide loading content (PLC) of nanoparticles was investigated by high performance liquid chromatography (HPLC; 1260 Infinity, Agilent Company, California, USA) using a C18 column (5 $\mu \mathrm{m}, 4.6 \mathrm{~mm} \times 150 \mathrm{~mm}$, Agilent Technologies; Santa Clara, CA, USA) at room temperature. An appropriate aliquot of nanoparticles was dispersed in $\mathrm{CH}_{2} \mathrm{Cl}_{2}(5 \mathrm{ml})$ and sonicated for $5 \mathrm{~min}$, followed by evaporation of the organic solvent at room temperature. Then abamectin/azoxystrobin was diluted to an appropriate volume with methanol. The mobile phase was composed of methanol and water

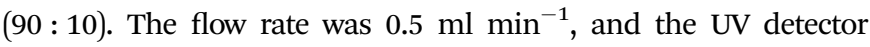
wavelength was $245 \mathrm{~nm}$.

\section{Particle size, polydispersity index and morphological characterization of nanoparticles}

The hydrodynamic particle size and polydispersity index (PDI) of nanoparticles were investigated by dynamic light scattering (DLS; Zetasizer Nano-ZS90, Malvern, Worcestershire, UK). The average value of three measurements was adopted. A scanning electron microscope (SEM; JSM-7401 F, JEOL Ltd., Akishimashi, Japan) and a transmission electron microscope (TEM; HT7700, Hitachi, Tokyo, Japan) were used to visualize the morphological characterizations of nanoparticles. An aliquot $(10 \mu \mathrm{l})$ of the re-dispersed nanoparticles was dropped on the surface of a cleaned silicon slice and coated with gold (thickness $\leq 2 \mathrm{~nm}$ ) after drying at room temperature. The SEM images were recorded at $5 \mathrm{kV}$ and the work distance was $8.5 \mathrm{~mm}$. For TEM, $6 \mu \mathrm{l}$ of the dispersed nanoparticles was dropped on the surface of a cleaned copper grid. The TEM images were performed at $80 \mathrm{kV}$ and $10 \mu \mathrm{A}$ after the nanoparticles were completely dried.

\section{Determination of the sustained release behavior of nanoparticles}

The sustained release behavior of abamectin/azoxystrobin nanoparticles was investigated by HPLC. Five $\mathrm{mg}$ of the active abamectin and $10 \mathrm{mg}$ of the nanoparticles were each suspended in $60 \%$ methanol solution $(5 \mathrm{ml})$ and the suspensions were carefully transferred to different dialysis bags (2000 MWCO). The dialysis bags were sealed tightly and placed into $60 \%$ methanol solution $(95 \mathrm{ml}$ ) in brown bottles that were used as the release media. The released abamectin/azoxystrobin was determined by collecting $5 \mathrm{ml}$ aliquots of the release media 

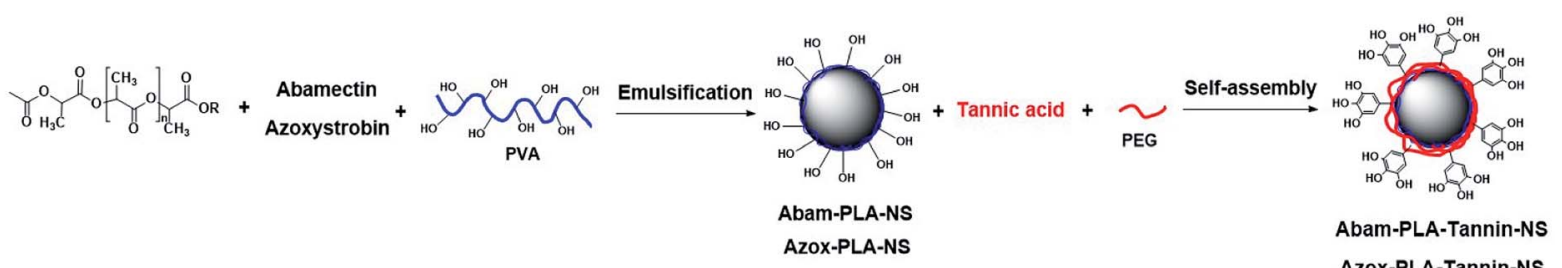

Scheme 1 Schematic illustration of the preparation of Abam-PLA-Tannin-NS and Azox-PLA-Tannin-NS.

outside the dialysis bags after specific time. The concentration was determined from the calibration curve by measuring the peak area of each aliquot at $245 \mathrm{~nm}$ using the HPLC method as described above. The accumulated release percentages were determined against time.

\section{Determination of the photodegradation behavior of nanoparticles}

Active abamectin and nanoparticles (100 $\mathrm{mg}$ each) were irradiated by UV light in a light incubator (XT5409-XPC80, $400 \mathrm{~W}$, Xutemp Technic Apparatus Co., Ltd., China). All of samples rotated around the light at a $10 \mathrm{~cm}$ distance and $25^{\circ} \mathrm{C}$. Samples $(5 \mathrm{mg})$ were collected after 12, 24, 48, 72 and $96 \mathrm{~h}$. The photodegradation behavior of abamectin at specific time intervals was measured by HPLC as described above.

\section{Wettability of nanoparticles on live cucumber foliage}

The wettability test was determined based on contact angle. Cucumber plants were selected 21 days after seeding from a light incubator. All dust on the foliage surface was carefully removed to prevent damage to the foliage structure. The foliage parts were carefully cut and adhered to glass slides. The measurements were performed with a contact angle (CA) instrument (JC2000D2M, Zhongchen Digital Technic Apparatus Company, Ltd., China); aqueous solutions of nanoparticles (3 $\mu \mathrm{l})$ were dropped onto the foliage surface. The average value of five measurements was adopted.

\section{Retention rate of nanoparticles on live cucumber foliage}

The retention test was determined based on SEM images. Cucumber plants were selected 21 days after seeding from a light incubator. All dust on the foliage surface was carefully removed to

Table 1 Mean size, polydispersity index (PDI), and pesticide loading content (PLC) of Abam-PLA-NS, Abam-PLA-Tannin-NS, Azox-PLA-NS and Azox-PLA-Tannin-NS

\begin{tabular}{llll}
\hline Samples & $\begin{array}{l}\text { Hydrodynamic size } \\
(\mathrm{nm}) \pm \text { S.D. }\end{array}$ & PDI \pm S.D. & PLC \\
\hline Abam-PLA-NS & $240.7 \pm 1.9$ & $0.03 \pm 0.02$ & $46.9 \%$ \\
Abam-PLA-Tannin-NS & $243.6 \pm 1.2$ & $0.02 \pm 0.01$ & $38.9 \%$ \\
Azox-PLA-NS & $249.3 \pm 4.6$ & $0.03 \pm 0.03$ & $33.8 \%$ \\
Azox-PLA-Tannin-NS & $256.7 \pm 0.6$ & $0.05 \pm 0.03$ & $30.5 \%$
\end{tabular}

prevent damage to the foliage structure. Nanoparticles samples (500 $\mu \mathrm{l}, 3.0 \mathrm{mg} \mathrm{ml}{ }^{-1}$ ) were sprayed onto the surfaces of cleaned cucumber foliage at a distance of $15 \mathrm{~cm}$. The treated cucumber foliage was dried for $4 \mathrm{~h}$ under vacuum after drying at ambient temperature, and then further SEM measurements were carried out at $3 \mathrm{kV}$. At the same time, control tests were conducted in which foliage samples after spraying and drying at room temperature and under vacuum were continually washed with deionized water (100 $\mathrm{ml})$.

\section{Determination of the biological efficacy of nanoparticles}

The indoor toxicity of Abam-PLA-Tannin-NS, Abam-PLA-NS and two kinds of commercial WDGs were evaluated using the leaf-dip method. Fresh cabbage (Brassica oleracea L.) leaves with a diameter of around $6 \mathrm{~cm}$ were fully immersed in aqueous solutions of 4 kinds of abamectin test samples with $0.78125,1.625,3.125,6.25$, 12.5, 25 and $50 \mathrm{ppm}$ containing $0.1 \%$ Triton X-100 for 10 s. Each treated leaf was dried at room temperature and placed in a culture dish; 20 similar active aphids (Myzus persicae L.) were introduced into each dish. The dishes were sealed with microporous plastic wrap and incubated at $75 \%$ humidity, $25{ }^{\circ} \mathrm{C}$ and $16 \mathrm{~h}: 8 \mathrm{~h}$ (light: dark) cycle. Mortality of aphids was counted after $48 \mathrm{~h}$. The regression equation, median lethal concentration $\left(\mathrm{LC}_{50}\right)$ and its 95\% confidence interval were calculated using DPS v12.01 statistical software. Each experiment was repeated four times and the average value was adopted.

The formulations of Azox-PLA-NS, Azox-PLA-Tannin-NS, commercialize microcapsule and SC were accurately diluted with sterile melted PDA medium to the final concentrations: $0.1,0.5,1,5,10$ and $20 \mathrm{ppm}$. Equal volumes of sterile deionized water were added into the sterile melted PDA medium as the control check (CK) group. The Fusarium was inoculated onto PDA at $27 \pm 1{ }^{\circ} \mathrm{C}$ for 6 days. The diameter of mycelium growth was measured by criss-cross method. The toxicity regression equations and $\mathrm{EC}_{50}$ were calculated using SPSS 2.0 statistical software. Each experiment was implemented in triplicate.

\section{Results and discussion}

Particle size, polydispersity index (PDI), pesticide loading content (PLC), and morphological characterization of nanoparticles

The experimental results of Abam-PLA-NS, Abam-PLA-TanninNS, Azox-PLA-NS and Azox-PLA-Tannin-NS on the mean size, PDI and PLC were summarized in Table 1 . The hydrodynamic 
sizes of Abam-PLA-NS and Azox-PLA-NS measured by DLS were 240.7 and $249.3 \mathrm{~nm}$, and they increased to 243.6 and $256.7 \mathrm{~nm}$ for the Abam-PLA-Tannin-NS and Azox-PLA-Tannin-NS preparations, respectively (Table 1 ). These results suggested that the surface of Abam-PLA-NS and Azox-PLA-NS was covered with TA. The low PDIs of these nanopesticides were $<0.1$, implying a narrow size distribution and excellent monodispersion. According to the HPLC analytical results, the drug loading contents of Abam-PLA-NS and Azox-PLA-NS were $46.9 \%$ and $33.8 \%$, and they decreased to $38.9 \%$ and $30.5 \%$ for the AbamPLA-Tannin-NS and Azox-PLA-Tannin-NS preparations, respectively (Table 1), suggesting that TA was on the surface of nanoparticles. SEM imaging showed that these nanoparticles presented nearly uniform spheres, and the statistical average sizes of 100 nanoparticles from the SEM images were around $150 \mathrm{~nm}$, in good agreement with the DLS results (Fig. 1).

\section{The stability of Abam-PLA-NS and Abam-PLA-Tannin-NS under different storage conditions}

The stability of pesticides is very important. The DLS mean size and PDI were adopted to evaluate the storage stability of these nanopesticides at different temperatures $\left(0^{\circ} \mathrm{C}, 25^{\circ} \mathrm{C}\right.$ and $\left.54{ }^{\circ} \mathrm{C}\right)$. The mean size and PDI presented negligible variation at $0{ }^{\circ} \mathrm{C}$ and $25^{\circ} \mathrm{C}$, indicating that these nanopesticides were very stable at low temperature (Fig. 2). However, the mean size and PDI increased at $54{ }^{\circ} \mathrm{C}$, presumably because it is very close to the glass state temperature of PLA $\left(55^{\circ}\right)$. SEM images showed better observation of these changes (Fig. 3). The morphology of these nanopesticides was maintained and the distribution was monodispersed at $0{ }^{\circ} \mathrm{C}$ and $25{ }^{\circ} \mathrm{C}$. They became broken and aggregated at $54{ }^{\circ} \mathrm{C}$, implying that these nanopesticides were not stable at high temperature. (a)

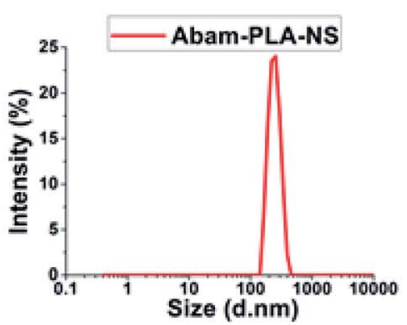

(d)

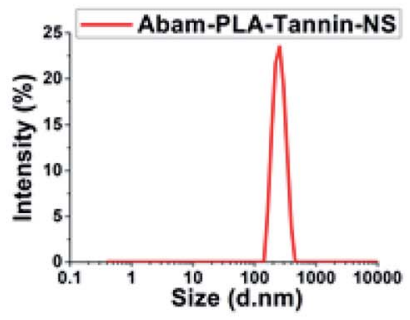

(g)

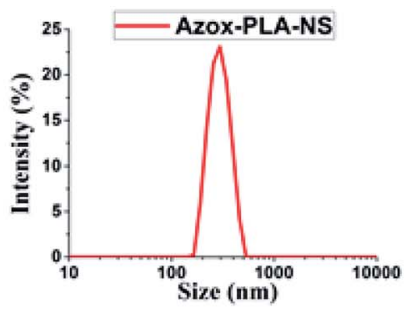

(j)

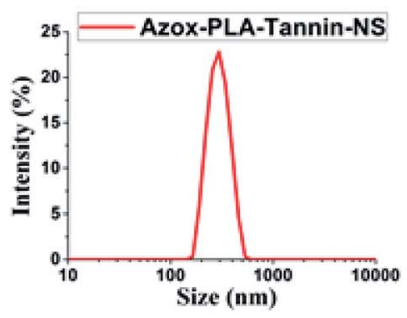

(b)

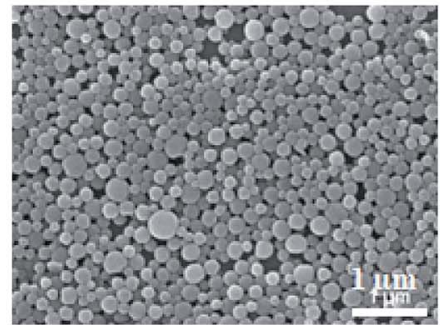

(e)

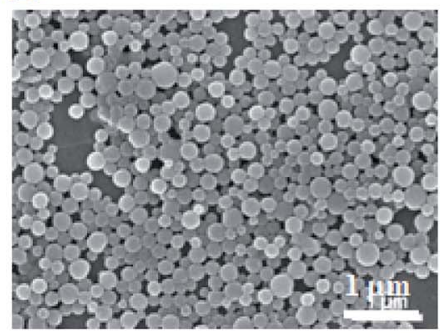

(h)

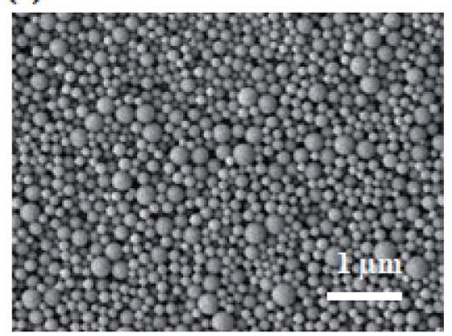

(k)

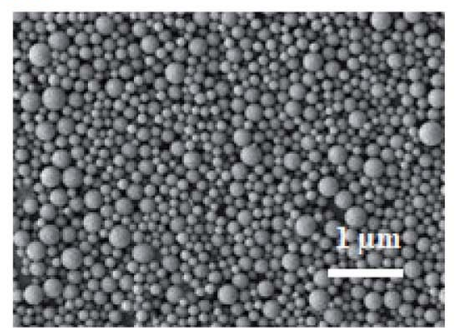

(c)

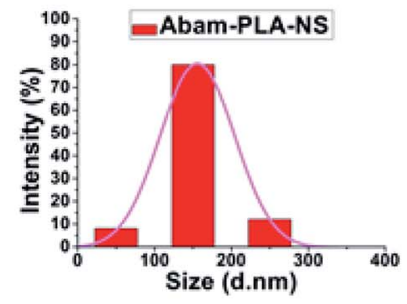

(f)

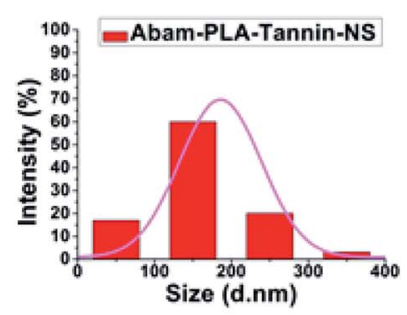

(i)

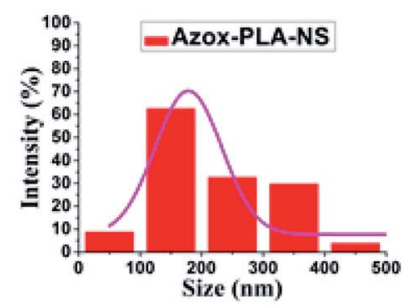

(I)

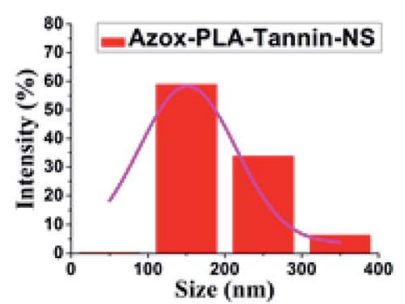

Fig. 1 Hydrodynamic size, scanning electron microscopy (SEM) images, and size distributions of Abam-PLA-NS (a-c), Abam-PLA-Tannin-NS $(\mathrm{d}-\mathrm{f})$, Azox-PLA-NS ( $\mathrm{g}-\mathrm{i})$ and Azox-PLA-Tannin-NS (j-l). The scale bar in SEM images is $1.0 \mu \mathrm{m}$. 

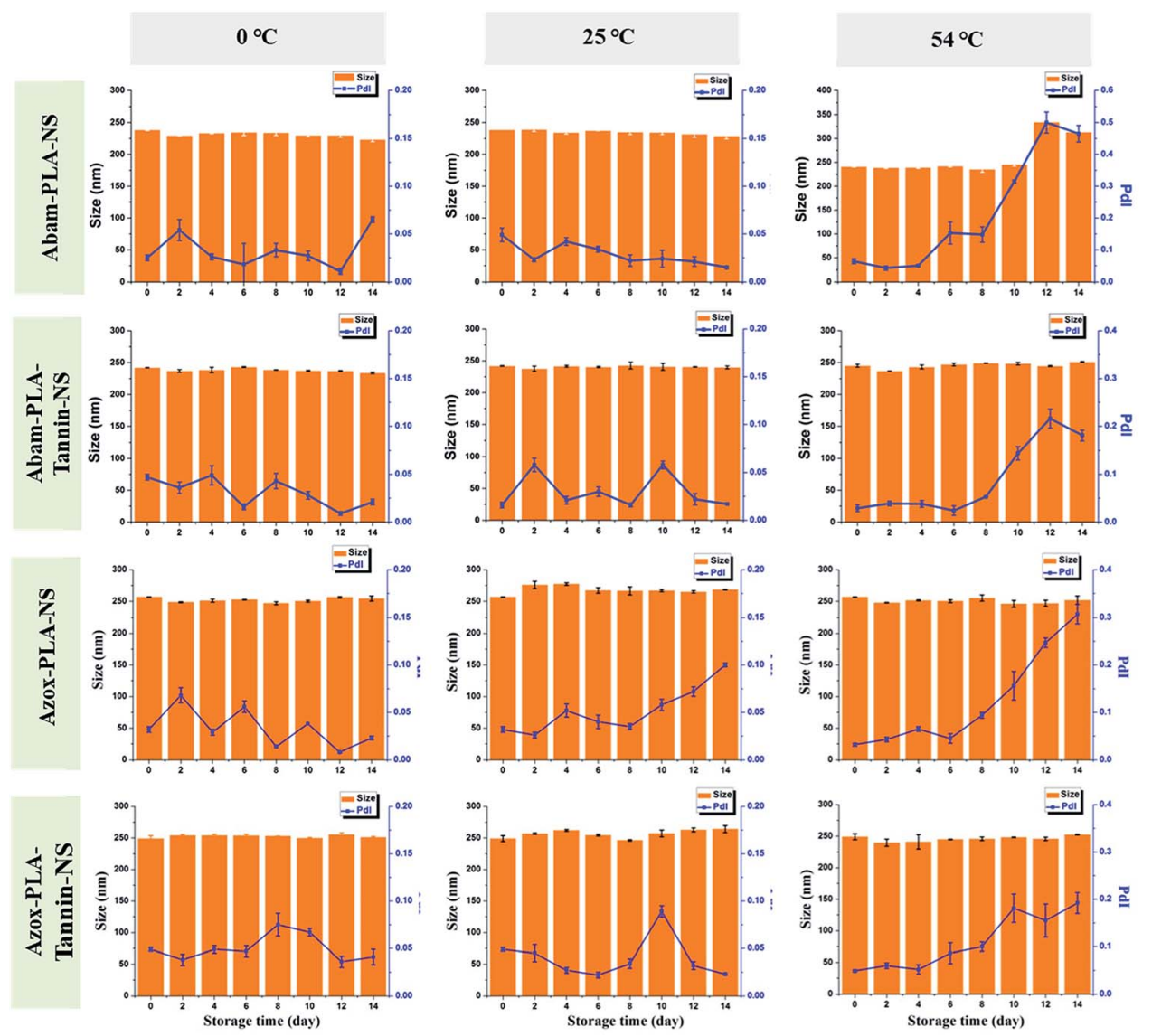

Fig. 2 Time dependent variation of dynamic light scattering mean size and polydispersity index (PDI) of Abam-PLA-NS, Abam-PLA-Tannin-NS, Azox-PLA-NS and Azox-PLA-Tannin-NS at different temperatures.

\section{The sustained release and photodegradation behavior of} nanoparticles

The prepared nanopesticides exhibited sustained release behavior when compared with active abamectin and azoxystrobin. The sustained release profiles and fitting equations of active abamectin, azoxystrobin and nanopesticides were presented in Fig. 4. As a result, Abam-PLA-NS, Abam-PLA-TanninNS, Azox-PLA-NS and Azox-PLA-Tannin-NS exhibited slower release rates than that of active abamectin and azoxystrobin, possessing the favorable sustained release behavior. The release rates of active abamectin and azoxystrobin were relatively fast, and they were completely released within $24 \mathrm{~h}$. In contrast, the release rates of Abam-PLA-NS, Abam-PLA-Tannin-NS, Azox-PLANS and Azox-PLA-Tannin-NS were gradual, with sustained release over $>120 \mathrm{~h}$. These results indicated that nanoparticles as a carrier could prolong the leaching time and increase the utilization efficiency of pesticides, resulting in reduced environmental residues and pollution. The sustained release profiles of the two types of nanopesticides were quite similar, implying that the TA added into Abam-PLA-NS and Azox-PLA-NS had negligible differential effects on the release behavior of abamectin and azoxystrobin. The accumulated release curves showed first-order kinetics, with $R^{2}$ values over 0.99 .

Abamectin is very sensitive to ultra violet (UV) light irradiation, and encapsulation was considered as an effective way to improve the photostability. The time-dependent response curves of the photodegradation percentage of active abamectin, Abam-PLA-NS and Abam-PLA-Tannin-NS were illustrated in Fig. 5. The photodegradation rate of active abamectin was relatively fast, with around $50 \%$ decomposing after $48 \mathrm{~h}$ of continuous UV irradiation. In contrast, much lower amounts of the abamectin loaded in the nanoparticles (Abam-PLA-NS and Abam-PLA-Tannin-NS) had decomposed within the same time period. These results indicated that the photostability of abamectin loaded in the nanoparticles was substantially improved.

\section{Wettability and retention of nanoparticles on crop foliage}

The wettability and retention of pesticides on the surface of crop foliage are very important to enhance deposition, adsorption, adhesion and utilization efficiency. It is well known that the complex microstructure on the rough foliage surface, such as the wax layer, tomenta, nervure and stomata, influence the wettability and retention properties with external objects. The surface of cucumber foliage has hydrophobic waxy composition, which prevents droplets from contacting the surface of foliage. The CA is an essential index to evaluate the wettability of pesticide formulations and the CA optical images on cucumber foliage were shown in Fig. 6. The mean CA values of Abam-PLA-NS, Abam-PLA-Tannin-NS, Abam-PLA-NS and AzoxPLA-Tannin-NS on cucumber foliage were $93.3^{\circ}, 91.0^{\circ}, 94.0^{\circ}$, 


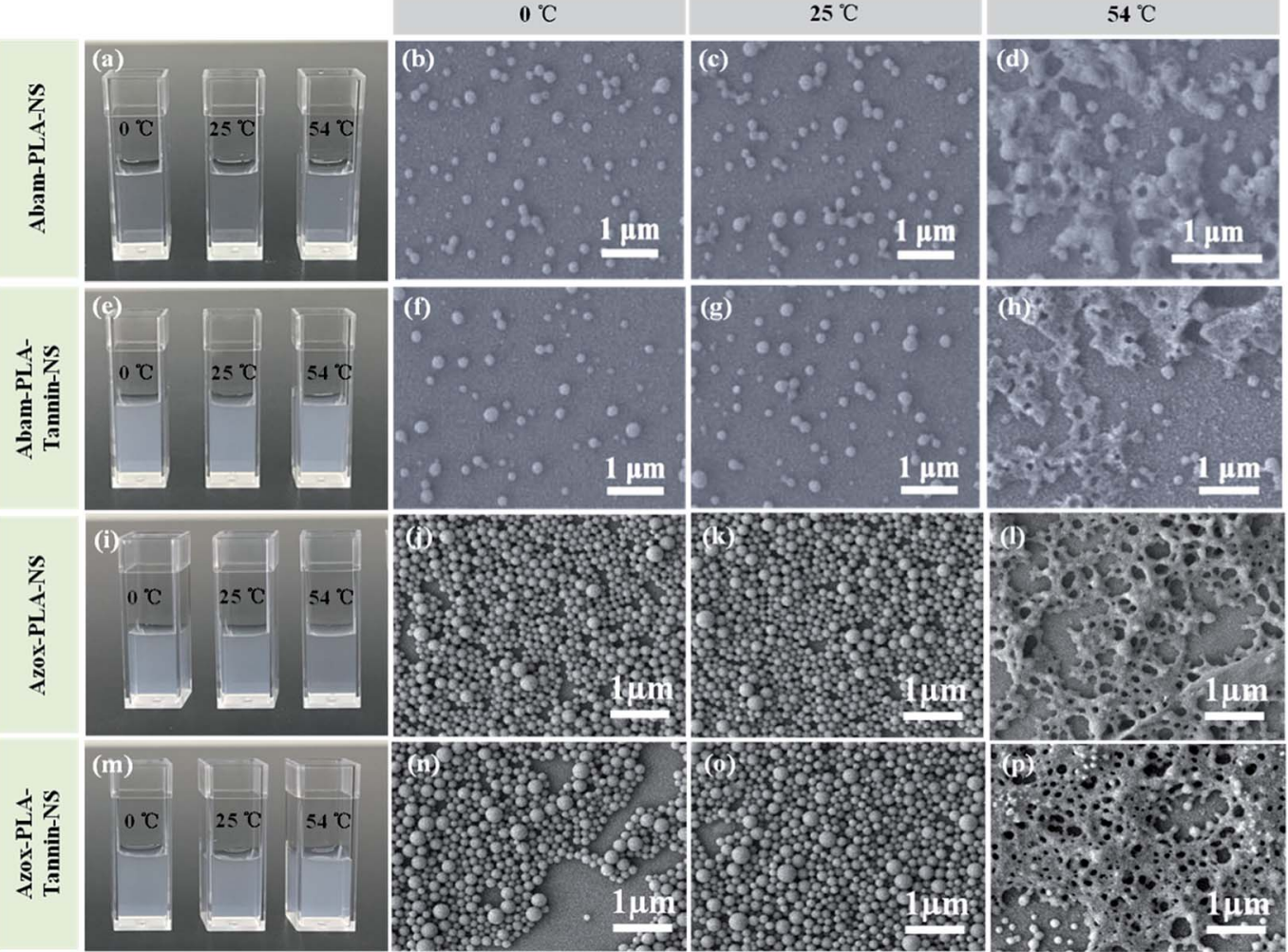

Fig. 3 Photographs and scanning electron microscopy (SEM) images of Abam-PLA-NS, Abam-PLA-Tannin-NS, Azox-PLA-NS and Azox-PLATannin-NS at different temperatures after 14 days storage. The scale bar in SEM images is $1.0 \mu \mathrm{m}$.

and $91.5^{\circ}$, respectively (Fig. 6), indicating that TA on the surface of nanopesticides could slightly improve wettability on crop foliage.

It is extremely hard to apply pesticides directly to harmful organisms. The crop foliage is the medium through which the pesticide activity is available to diseases and pests. The retention time of pesticides on crop foliage is very important to increase utilization efficiency. The retention time is highly related to the adhesion of pesticides to crop foliage. Here, the retention rate of pesticides by washing was adapted to roughly evaluate the adhesive force and retention time on crop foliage. HPLC was used to determine the pesticides concentration and retention rates. The calculated retention rates on cucumber foliage were $43 \%, 67 \%$, $31 \%$, and $27 \%$ for Abam-PLA-NS, Abam-PLA-Tannin-NS, AbamWDG-A and Abam-WDG-B, respectively (Fig. 7). The retention
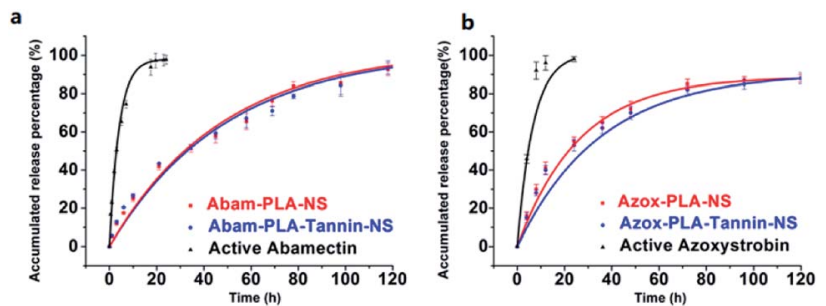

Fig. 4 (a) Sustained release profiles of active abamectin, Abam-PLANS and Abam-PLA-Tannin-NS. (b) Sustained release profiles of active azoxystrobin, Azox-PLA-NS and Azox-PLA-Tannin-NS. The accumulated release percentages were obtained against time from the sustained release amounts. rates on cucumber foliage were $38 \%, 59 \%, 35 \%$, and $27 \%$ for AzoxPLA-NS, Azox-PLA-Tannin-NS, SC and microcapsule, respectively. When compared with Abam-PLA-NS and Azox-PLA-NS, Abam-PLATannin-NS and Azox-PLA-Tannin-NS showed much greater affinity to cucumber foliage, with more than $50 \%$ enhancement. The TA on the surface of nanopesticides could remarkably increase the adhesion to cucumber foliage, because of the polyphenol groups enhancing the adhesive binding to the crop foliage surfaces. These results are in agreement with previous reports of polyphenol adhesive chemistry. ${ }^{37,38}$ To better visualize the variation before and after washing in spatial dimensions, SEM was applied to characterize the deposition and retention behavior of nanoparticles on

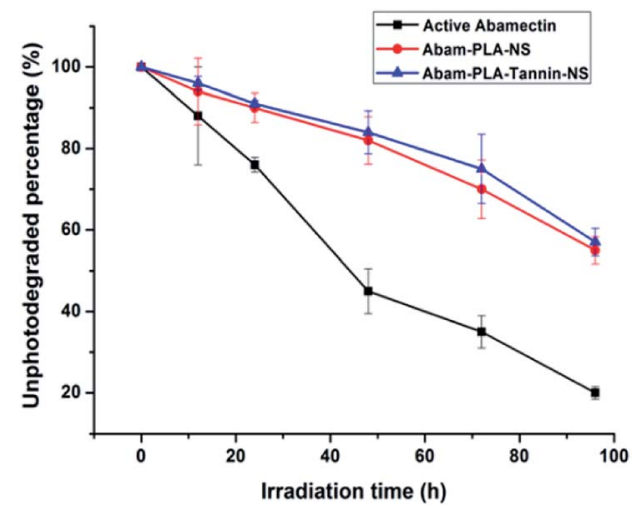

Fig. 5 Photostability curves of active abamectin, Abam-PLA-NS and Abam-PLA-Tannin-NS versus irradiation time at $25^{\circ} \mathrm{C}$. 
(a)

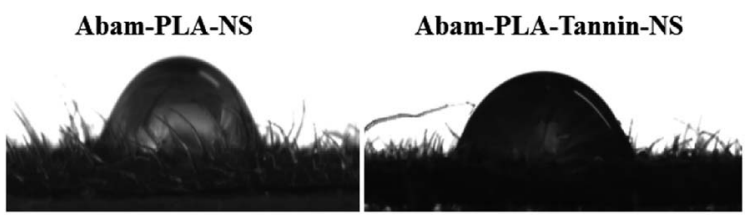

(c) Azox-PLA-NS

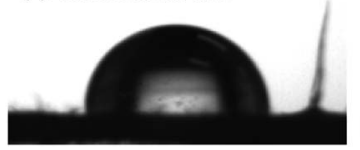

(d) Azox-PLA-Tannin-NS

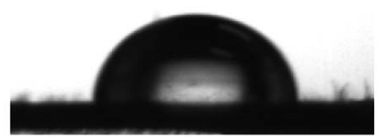

Fig. 6 Contact angles of Abam-PLA-NS (a), Abam-PLA-Tannin-NS (b), Abam-PLA-NS (c) and Azox-PLA-Tannin-NS (d) on the surface of cucumber foliage.
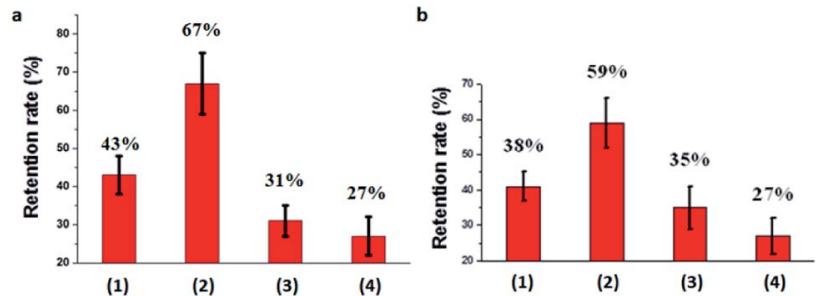

Fig. 7 (a) Retention rates on the cucumber foliage surface of Abam-PLANS (1), Abam-PLA-Tannin-NS (2) and commercially available formulations Abam-WDG-A (3) and Abam-WDG-B (4). (b) Retention rates on the cucumber foliage surface of Azox-PLA-NS (1), Azox-PLA-Tannin-NS (2), and commercially available formulations SC (3) and microcapsule (4).

the surface of cucumber foliage. There were many more observed particles of Abam-PLA-Tannin-NS than that of Abam-PLA-NS on the surface of cucumber foliage after washing, confirming that
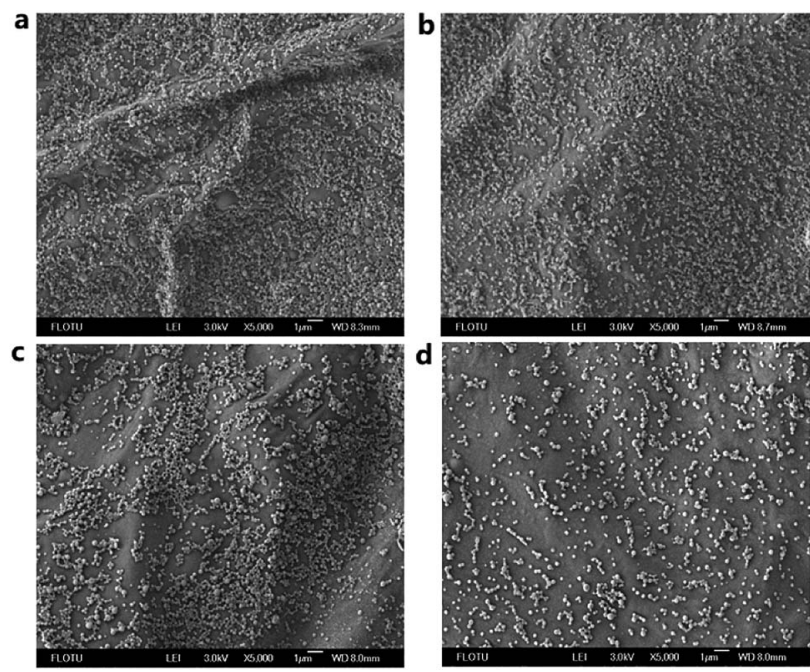

Fig. 8 Scanning electron microscopy images of Abam-PLA-NS and Abam-PLA-Tannin-NS on the surface of cucumber foliage. (a) Image of Abam-PLA-Tannin-NS on the surface of cucumber foliage, (b) image of Abam-PLA-NS on the surface of cucumber foliage, (c) image of Abam-PLATannin-NS after washing, and (d) image of Abam-PLA-NS after washing.
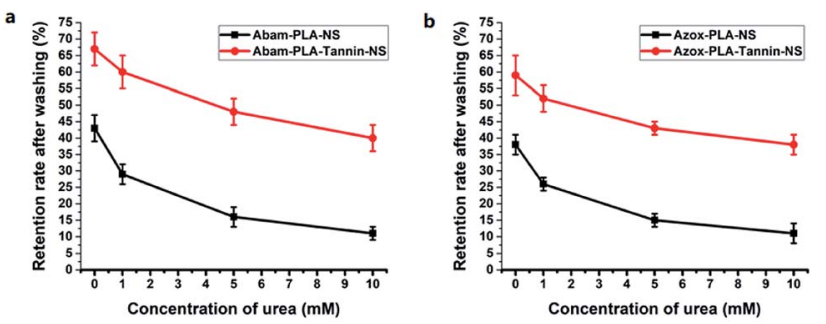

Fig. 9 The retention rates effects of different urea concentration on the cucumber foliage surface with Abam-PLA-NS, Abam-PLA-TanninNS (a), Azox-PLA-NS and Azox-PLA-NS (b).

Abam-PLA-Tannin-NS had better adhesion to cucumber foliage than that of Abam-PLA- NS (Fig. 8).

To gain insight into the interaction mechanism between nanopesticides and cucumber foliage, urea (a strong hydrogen bond disrupting agent) was used as washing solvent to evaluate the change in retention rate. The retention rates of Abam-PLANS, Abam-PLA-Tannin-NS, Azox-PLA-NS and Azox-PLA-TanninNS decreased in the presence of urea, and they were urea concentration-dependent (Fig. 9). The retention rates of AbamPLA-NS and Azox-PLA-NS were more sensitive to the urea concentration when compared with those of Abam-PLA-TanninNS and Azox-PLA-Tannin-NS. These results confirmed that the interaction force between nanoparticles and cucumber foliage was mainly from hydrogen bonding, and Abam-PLA-Tannin-NS and Azox-PLA-Tannin-NS showed strong binding to the foliage surface. In addition, there are also possible coordinate bonds between Abam-PLA-Tannin-NS and Azox-PLA-Tannin-NS and the crop foliage, because polyphenols can easily coordinate many metal ions. These multimodal bindings between AbamPLA-Tannin-NS and Azox-PLA-Tannin-NS and the foliage surface result in the strong adhesive attraction between them.

\section{Biological efficacy}

In this study, the leaf dipping method was used to determine the indoor toxicity of Abam-PLA-NS, Abam-PLA-Tannin-NS and commercial formulations (Abam-WDG-A and Abam-WDG-B).

Table 2 Indoor toxicity results of abamectin formulations

\begin{tabular}{llll}
\hline Formulation & Toxicity regression equation & $R^{2}$ & $\mathrm{LC}_{50}(\mathrm{ppm})$ \\
\hline Abam-PLA-NS & $y=3.54+1.18 x$ & 0.97 & 17.38 \\
Abam-PLA-Tannin-NS & $y=3.52+1.43 x$ & 0.93 & 10.68 \\
Abam-WDG-A & $y=3.46+0.92 x$ & 0.83 & 47.29 \\
Abam-WDG-B & $y=3.64+0.90 x$ & 0.92 & 32.58
\end{tabular}
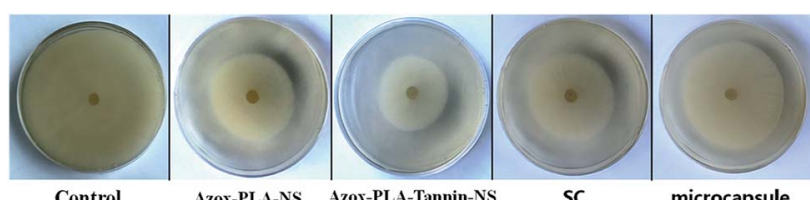

Fig. 10 The antifungal activity of different formulations (10 ppm/6 days). 
Table 3 Antifungal activities of four azoxystrobin formulations

\begin{tabular}{llrr}
\hline Sample & Toxicity regressive equation & $R^{2}$ & LC $_{50}\left(\mu \mathrm{g} \mathrm{ml}^{-1}\right)$ \\
\hline Azox-PLA-NS & $y=-0.41+0.14 x$ & 0.945 & 6.53 \\
Azox-PLA-Tannin-NS & $y=-0.35+0.14 x$ & 0.938 & 4.67 \\
$25 \%$ SC & $y=-0.21+0.25 x$ & 0.965 & 6.97 \\
$10 \%$ microcapsule & $y=-0.50+0.10 x$ & 0.914 & 12.35
\end{tabular}

The toxicity of pesticides to aphids (Myzus persicae L.) was shown in Table 2. The $\mathrm{LC}_{50}$ values were 17.38, 10.78, 47.29, and $32.58 \mathrm{ppm}$ for Abam-PLA-NS, Abam-PLA-Tannin-NS, AbamWDG-A and Abam-WDG-B, respectively (Table 2). The toxicity of Abam-PLA-Tannin-NS to aphids (Myzus persicae L.) was 1.6fold higher than that of Abam-PLA-NS, presumably because of higher pesticide retention on the surface of cucumber foliage in the leaf dipping experiment. These results agreed well with the foliar retention results. The antifungal activities of Azox-PLANS, Azox-PLA-Tannin-NS, Commercial SC and microcapsule to Fusarium were evaluated (Fig. 10). The $\mathrm{LC}_{50}$ values were 6.53, 4.67, 6.97, and $12.35 \mu \mathrm{g} \mathrm{ml}{ }^{-1}$ for Azox-PLA-NS, Azox-PLATannin-NS, Commercial SC and microcapsule, respectively (Table 3). Azox-PLA-Tannin-NS exhibited the highest antifungal activity, and the antifungal ability of Azox-PLA-Tannin-NS to a Fusarium (Myzus persicae L.) was 1.4-fold that of Azox-PLA-NS, probably owing to the interaction of TA in Azox-PLA-Tannin-NS and Fusarium. These results indicated that Abam-PLA-TanninNS and Azox-PLA-Tannin-NS with enhanced adhesion had increased efficacy against their target organisms when compared with the other formulations tested.

\section{Conclusions}

In this study, Abam-PLA-Tannin-NS and Azox-PLA-Tannin-NS with improved adhesion to crop foliage were successfully fabricated by chemical modification on the surface of AbamPLA-NS and Azox-PLA-NS using TA. These nanoparticles were spherical with excellent monodispersion. The diameters of the TA-loaded nanoparticles were slightly increased and the drug contents were slightly decreased when compared with their PLA-NS counterparts, implying successful coating with TA. They showed excellent continuous sustained release, and the photostability of abamectin in Abam-PLA-Tannin-NS against UV light irradiation was highly improved. The adhesive force was mainly from hydrogen binding between TA and foliage. The affinitive bindings of Abam-PLA-Tannin-NS and Azox-PLATannin-NS to foliage surface resulted in high adhesion and long retention time. Foliar-adhesive nanopesticides could be considered as a resource-saving and environmentally-friendly pesticide formulation, to decrease spraying dosage and pollution in food and the environment.

\section{Conflicts of interest}

There are no conflicts to declare.

\section{Acknowledgements}

We thank National Key Project of Research and Development Plan Program of China (2017YFD0200900) and China Postdoctoral Science Foundation (2018M630234) for the financial support.

\section{Notes and references}

1 What is a pesticide?, U.S. Environmental Protection Agency, 2007.

2 M. G. Mogul, H. Akin, N. Hasirci, D. J. Trantolo, J. D. Gresser and D. L. Wise, Resour., Conserv. Recycl., 1996, 16, 289-320.

3 Agricultural Statistics, United States Department of Agriculture, United States Government Printing Office, Washington, DC, 1992.

4 F. Worek, M. Koller, H. Thiermann and L. Szinicz, Toxicology, 2005, 214, 182-189.

5 D. P. Eyer, Toxicol. Rev., 2003, 22, 165-190.

6 International Code of Conduct on the Distribution and Use of Pesticides, Food and Agriculture Organization of the United Nations, 2007.

7 A. Grube, D. Donaldson, T. Kiely and L. Wu, Pesticides Industry Sales and Usage, U.S. Environmental Protection Agency, 2011.

8 S. M. Bradberry, A. T. Proudfoot and V. J. Allister, Toxicol. Rev., 2004, 23, 65-73.

9 M. Eddleston, P. Eyer, F. Worek, F. Mohamed, L. Senarathna, L. vonMeyer, E. Juszczak, A. Hittarage, S. Azhar, W. Dissanayake, M. H. R. Sheriff, L. Szinicz, A. H. Dawson and N. A. Buckley, Lancet, 2005, 366, 1452-1459.

10 M. Jokanovic, Toxicol. Lett., 2009, 190, 107-115.

11 V. C. Schreiner, E. Szocs, A. K. Bhowmik, M. G. Vijver and R. B. Schafer, Sci. Total Environ., 2016, 573, 680-689.

12 E. Malaj, P. C. von der Ohe, M. Grote, R. Kuhne, C. P. Mondy, P. U. Polatera, W. Brack and R. B. Schäfer, Proc. Natl. Acad. Sci. U. S. A., 2014, 111, 9549-9554.

13 M. Nuruzzaman, M. M. Rahman, Y. Liu and R. Naidu, J. Agric. Food Chem., 2016, 64, 1447-1483.

14 B. Wang, J. L. Song, A. J. Zeng, Y. J. Liu, J. Zhang and X. K. He, Chin. J. Pestic. Sci., 2012, 14, 334-340.

15 X. Zhao, H. Cui, Y. Wang, C. Sun, B. Cui and Z. Zeng, J. Agric. Food Chem., 2018, 66, 6504-6512.

16 M. Kah, R. S. Kookana, A. Gogos and T. D. Bucheli, Nat. Nanotechnol., 2018, 13, 677-684.

17 M. Kah, H. Walch and T. Hofmann, Environ. Sci.: Nano, 2018, 5, 882-889. 
18 E. Worrall, A. Hamid, K. Mody, N. Mitter and H. Pappu, Agronomy, 2018, 8, 285.

19 Y. Xiang, G. Zhang, C. Chen, B. Liu, D. Cai and Z. Wu, ACS Sustainable Chem. Eng., 2018, 6, 1192-1201.

20 I. H. Hafez, A. R. Osman, E. A. Sewedan and M. R. Berber, J. Agric. Food Chem., 2018, 66, 8237-8245.

21 P. Zhao, W. Yuan, C. Xu, F. Li, L. Cao and Q. Huang, J. Agric. Food Chem., 2018, 66, 11592-11600.

22 X. Jia, W. Sheng, W. Li, Y. Tang, Z. Liu and F. Zhou, ACS Appl. Mater. Interfaces, 2014, 6, 19552-19558.

23 L. R. Khot, S. Sankaran, J. M. Maja, R. Ehsani and E. W. Schuster, Crop Prot., 2012, 35, 64-70.

24 M. Kah and T. Hofmann, Environ. Int., 2014, 63, 224-235.

25 M. L. Yu, J. W. Yao, J. Liang, Z. H. Zeng, B. Cui, X. Zhao, C. Sun, Y. Wang, G. Liu and H. Cui, RSC Adv., 2017, 7, 11271-11280.

26 H. Lee, S. M. Dellatore and W. M. Miller, Science, 2007, 318, 426-430.

27 P. K. Forooshani and B. P. Lee, Polym. Chem., 2017, 55, 9-33.

28 H. Lee, J. Rho and P. B. Messersmith, Adv. Mater., 2008, 21, 431-434.
29 A. Postma, Y. Yan, Y. Wang, A. N. Zelikin, E. Tjipto and F. Caruso, Chem. Mater., 2009, 21, 3042-3044.

30 C. Cheng, S. Li, S. Q. Nie, W. F. Zhao, H. Yang, S. D. Sun and C. Zhao, Biomacromolecules, 2012, 13, 4236-4246.

31 X. Xiong, Y. M. Liu, F. Shi, G. W. Zhang, J. Weng and S. X. Qu, J. Bionic Eng., 2018, 15, 461-470.

32 J. Liang, M. Yu, L. Guo, B. Cui, X. Zhao, C. Sun, Y. Wang, G. Liu, H. Cui and Z. Zeng, J. Agric. Food Chem., 2018, 66, 6578-6584.

33 N. S. Khan, A. Ahmad and S. M. Hadi, Chem.-Biol. Interact., 2000, 125, 177-189.

34 T. G. Shutava, S. S. Balkundi, P. Vangala, J. J. Steffan, R. L. Bigelow, J. A. Cardelli, D. P. O'Neal and Y. M. Lvov, ACS Nano, 2009, 3, 1877-1885.

35 K. M. Riedl and A. E. Hagerman, J. Agric. Food Chem., 2001, 49, 4917-4923.

36 B. Li, W. Wang, X. Zhang, D. Zhang, Y. Ren, Y. Gao, W. Mu and F. Liu, Adv. Funct. Mater., 2017, 27, 1701841.

37 K. Keumyeon, M. Shin, M. Koh, J. Ryu, M. Lee, S. Hong and H. Lee, Adv. Funct. Mater., 2015, 25, 2402-2410.

38 Y. Du, W. Qiu, Z. Wu, P. Ren, Q. Zheng and Z. Xu, Adv. Mater. Interfaces, 2016, 3, 1600167. 